\title{
Présentation
}

L'enseignant : acteur ou spectateur du changement?

\section{Dominique Raulin}

\section{CpenEdition}

\section{Journals}

Édition électronique

URL : https://journals.openedition.org/ries/1301

DOI : $10.4000 /$ ries. 1301

ISSN : 2261-4265

Éditeur

France Education international

Édition imprimée

Date de publication : 1 décembre 2005

Pagination : 83-84

ISSN : 1254-4590

Référence électronique

Dominique Raulin, «Présentation », Revue internationale d'éducation de Sèvres [En ligne], 40 | décembre 2005, mis en ligne le 17 novembre 2011, consulté le 05 juillet 2021. URL : http://

journals.openedition.org/ries/1301; DOI : https://doi.org/10.4000/ries.1301

(c) Tous droits réservés 


\section{L'enseignant : acteur ou spectateur du changement?}

\section{Dominique Raulin}

La plupart des systèmes d'éducation, au-delà de leurs organisations administratives, connaissent des mutations et des difficultés analogues, depuis une trentaine d'années

Indépendamment de ces changements, les professeurs ont fait face également aux exigences toujours plus fortes des parents ou de la société. D’une certaine façon, ils ont été amenés à fournir au quotidien des réponses aux incohérences ou aux paradoxes de la société, sans pouvoir toujours se prévaloir, dans leurs choix, de décisions réglementaires.

Ainsi, le métier d'enseignant de 2005 n'a plus beaucoup de points communs avec celui du début des années 1980 : le public a changé, les finalités sont remises en cause (instruire ou éduquer?), les outils et les méthodes ne sont plus les mêmes, le rythme des changements des contenus d'enseignement s'est accéléré.

Ainsi se dessine, dans la société, l'image d'un corps enseignant conservateur, hostile aux changements, pessimiste, nostalgique d'un âge d'or où tous les élèves apprenaient, comprenaient et réussissaient (Espagne, Suisse, France)! Des interrogations sur la possibilité réelle de réformer l'éducation sont régulièrement formulées. Les corps enseignants seraient des corps immobiles constitués d'une multitude d'éléments en perpétuel mouvement.

Si les enseignants s'impliquent peu dans les processus de réformes, c'est, selon eux, qu'ils s'estiment mal informés sur les changements et tenus à l'écart de la réelle prise de décision (Roumanie, Espagne, Suisse). La défaillance du contrat moral entre eux et la nation, la survivance d'une organisation hybride croisant autonomie locale et contrôle de l'État (Angleterre), les carences en matière d'évaluation (France) les conduisent à prendre leur distance par rapport au changement.

La question fondamentale est donc de savoir si l'on peut s'accommoder de cette situation ou si elle est préjudiciable au bon fonctionnement et à la modernisation des systèmes éducatifs. Il ne faut toutefois pas s'égarer : il n'appartient pas aux enseignants de prendre individuellement des décisions qui relèvent de la responsabilité du pouvoir politique, exécutif et/ou législatif. Mais à l'inverse, on ne peut considérer qu'ils n'en sont pas partie prenante. En effet, les professeurs sont les acteurs du changement puisqu'ils ont pour mission de les mettre en œuvre dans les salles de classes : une innovation ou une réforme n'existe réellement que s'ils parviennent à l'appliquer. 
Au-delà de cette simple application, on peut s'interroger sur la participation des enseignants à la conception même et à la promotion d'une réforme : ne sont-ils pas les seuls à avoir l'expertise de la faisabilité à travers leur connaissance des élèves et de la réalité des classes? Cela suffit-il à en faire des experts du changement?

Une réforme ne peut pas être la simple généralisation d'une innovation menée par certains enseignants plus ou moins imaginatifs, audacieux ou chevronnés : en effet, les innovations ne sont souvent pas évaluées; la part de l'enseignant dans leur réussite est difficile à mesurer; et enfin, les conditions d'une extension réussie sont mal cernées.

Le professeur est un praticien avec tout le savoir-faire et l'expertise que cela induit, mais il n'est pas, au moins sur le plan statutaire, un chercheur en sciences de l'éducation, en pédagogie ou en didactique, encore moins en organisation systémique. Il ne dispose donc pas des éléments théoriques nécessaires pour concevoir et promouvoir une réforme. La question est de savoir s'il doit le devenir.

À l'évidence, face à ces évolutions, l'ensemble des pays s'interroge sur la professionnalisation enseignante (Suisse, Angleterre, Roumanie, France), sur la nécessité de redéfinir l'identité des enseignants, de se mettre d'accord sur le type de professeur dont la société a besoin (Espagne).

\section{ÉTUDES DE CAS :}

Roumanie, Espagne, Suisse, Angleterre, France 


\section{Roumanie}

\section{Attendre ou produire le changement?}

\section{Emil Paun}

La réforme scolaire roumaine a été réalisée dans un contexte d'urgence, de rupture socio-politique, d'anomie (au moins pendant la première période). On peut comparer cette rupture avec un changement de paradigme.

La pré-réforme (1990-1991) visait à des changements immédiats et urgents des contenus d'enseignement : élimination des disciplines profondément politisées, désidéologisation d'autres disciplines (l'histoire, la littérature, la philosophie, etc.). Au niveau de la structure du système scolaire, le début de la scolarité est passé de six à sept ans, la durée de la scolarité obligatoire de dix à huit années. Pendant cette période, la cohérence des changements et le degré de participation des enseignants ont été très faibles.

Pour la préparation de la réforme (1992-1994), les autorités roumaines ont commencé à négocier avec la Banque mondiale pour un accord sur le financement de la réforme scolaire. Pendant ces négociations, les objectifs et les composantes de la réforme ont été définis. Cet accord, plutôt pragmatique, définissait une politique de réformes et non une philosophie de la réforme. Il s'agissait de réformer le curriculum et les manuels scolaires, le système de la formation des enseignants, le système d'évaluation des élèves, le management et la gestion des écoles.

Une première constatation rétroactive : pendant cette période, les autorités roumaines n'ont pas eu toujours un rôle d'acteur des changements proposés.

\section{La réforme de 1995}

En 1995, la loi de l'enseignement qui établissait le cadre juridique de la réforme scolaire a été adoptée. À partir de ce moment, on a essayé d'impliquer et de mobiliser davantage les enseignants en tant qu'acteurs de la réforme. Comme les différents axes de la réforme ont été définis en coopération avec des acteurs différents - des groupes d'experts américains, français, anglais -, un relatif manque de cohérence en a résulté, surtout dans les premières années. Les messages qui parvenaient au niveau des établissements étaient parfois contradictoires, ce qui a entraîné un manque d'engagement de la part des enseignants.

Dans ce contexte, la réforme a été projetée et réalisée (au début) du haut vers le bas, sur le modèle centre/périphérie. Les enseignants n'ont donc pas été sollicités pour participer aux prises de décisions. Ils ont plutôt été 
spectateurs de la réforme. Était-il possible d'agir autrement? Je ne le crois pas. Cette situation s'explique par de nombreux facteurs :

- la complexité, l'amplitude et la radicalité des changements;

- la quasi-absence d'une culture de la participation due au fait qu'avant 1990, tout était centralisé, contrôlé et dirigé; le comportement dominant de la population a été le conformisme social;

- l'inquiétude, la prudence et même la résistance des enseignants face aux changements trop rapides et trop radicaux, dans un contexte d'instabilité et de manque de certitudes de la vie quotidienne;

- l'existence d'un syndrome qu'on pourrait appeler la «dépendance du centre».

On peut ajouter aussi que la réforme scolaire a été pensée dans un paradigme plutôt normatif, rationaliste et technologique qui n'était pas très stimulant pour le développement du comportement participatif des enseignants.

\section{Une identité professionnelle à construire}

On peut parler du rôle d'acteur des enseignants soit au niveau individuel soit au niveau du groupe socioprofessionnel. En ce qui nous concerne, notre analyse portera sur le groupe socioprofessionnel car l'implication active des enseignants dans les changements et leur capacité à les influencer dépend de leur force en tant que groupe, de leur sentiment identitaire.

L'une des variables de l'identité professionnelle des enseignants est la composition du corps enseignant. Hormis sa féminisation croissante (présente presque dans tous les pays et dont on ne cherchera pas ici à analyser les causes), quelques aspects particuliers à la Roumanie peuvent être identifiés.

Premièrement, un relatif manque d'unité et d'homogénéité de la structure sociale du corps enseignant. Il n'y a pas de bassin social stable et homogène de recrutement pour la carrière enseignante. Appartenance sociale hétérogène, culture hétérogène.

Deuxièmement, le phénomène de vieillissement est évident. Les enseignants âgés sont plus nombreux que les jeunes. Cette situation est due en partie au fait que cette profession n'est pas très attractive actuellement (salaires bas, statut et prestige social dévalorisés, etc.). Les jeunes qui terminent leurs études universitaires préfèrent des professions mieux payées et plus prestigieuses, surtout dans le secteur privé. Une autre cause est le manque chronique de personnel enseignant qualifié. Dans ces conditions, beaucoup d'enseignants âgés sont sollicités, même après leur départ à la retraite, pour continuer leur activité. Dans ce contexte, on observe une coupure entre le rapport au statut et le rapport au métier chez les enseignants. En général, même si le statut est dévalorisé, les enseignants, surtout les plus âgés, valorisent leur métier. 
On peut pointer un autre phénomène. À cause de la transition, de la réforme et de la restructuration économique, beaucoup de ceux qui avaient une qualification supérieure (surtout les ingénieurs) ont été mis au chômage. Parmi eux, beaucoup ont demandé à travailler comme professeurs. Mais ils n'ont pas de formation pédagogique et didactique et sont, eux aussi, plus âgés (quarante ans et plus).

Cette hétérogénéité fait que l'existence du corps enseignant en tant que groupe professionnel homogène est faible. Cela peut expliquer, en partie, les difficultés du corps enseignant à s'engager et à s'impliquer plus activement dans la réalisation de la réforme (surtout au début).

En ce qui concerne les critères de recrutement, il faut mentionner que, malheureusement, le recrutement des futurs enseignants sur une base professionnelle n'existe pas encore (sauf pour les enseignants du primaire). La formation initiale des professeurs du secondaire se fait dans différentes facultés : mathématiques, chimie, histoire, philosophie, psychologie, géographie, etc. Ceux qui désirent suivre une formation pour le métier enseignant peuvent suivre une option (à partir de la première année d'études) pour un module de psychopédagogie et didactique de la spécialité. Les études disciplinaires et les études de psychopédagogie et didactique se font dans le même temps, pendant quatre ans. Il n'y a pas de critères de sélection pour le module mentionné et il n'existe pas d'épreuves spéciales de certification professionnelle à la fin des études. C'est une fragilité supplémentaire pour l'identité professionnelle du corps enseignant.

Il est donc encore nécessaire de faire un travail de construction identitaire tant au niveau du statut, du prestige et de la reconnaissance sociale qu'au niveau de la professionnalisation du métier. Le corps enseignant en tant que groupe socioprofessionnel unifié reste, dans une grande mesure, à construire.

\section{Une culture d'acteur en train de se construire}

Pour les enseignants, être acteur des évolutions suppose différents degrés d'implication et de participation aux changements : participation à la prise de décision (au niveau central ou au niveau local) ainsi qu'à la mise en œuvre des décisions prises par divers groupes de décideurs.

En Roumanie, les enseignants participent plutôt à la mise en œuvre des décisions prises au niveau central mais, de plus en plus, ils sont sollicités pour s'impliquer au niveau des décisions prises localement par les écoles. Au niveau central, ils sont rarement consultés sur les changements à réaliser. Parfois, ils sont représentés dans différents groupes consultatifs du ministère, mais leur capacité d'influencer les décisions est faible.

Ces dernières années, une culture de la participation est en train de se construire au niveau du corps enseignant. Beaucoup d'enseignants se sont regroupés dans des associations professionnelles très actives dans les débats concernant la réforme scolaire. Ils mettent en place des projets de partenariat 
local pour le développement institutionnel des écoles. La création et la conduite de projets sont de plus en plus présentes dans la vie quotidienne des écoles. On observe un grand intérêt pour les différentes innovations ou expériences réussies d'enseignement et d'apprentissage, de didactique, etc. Au niveau local se sont mis en place des réseaux professionnels très actifs et des projets menés dans différents domaines à partir du développement institutionnel des écoles, en partenariat avec les parents et les autorités locales. Cela va jusqu'à l'activité avec les élèves au sein de la classe et dans l'espace social de l'école. Parallèlement à une culture d'acteur, un ethos de la participation, une culture professionnelle partagée sont en train de se développer. On observe, de plus en plus, un passage du statut d'exécutant au statut d'acteur.

\section{Difficultés actuelles}

Il existe encore des zones en difficulté, par exemple les zones rurales et la périphérie des grandes villes où les efforts des enseignants et des directeurs des écoles sont concentrés plutôt sur les moyens de survivre du point de vue financier et matériel. Il y a des communautés pauvres et, par conséquent, des écoles pauvres. Ces écoles se confrontent aussi au manque chronique d'enseignants qualifiés et stables.

La formation initiale des enseignants reste encore trop théorique et focalisée surtout sur la discipline à enseigner. Les élèves sont vus seulement comme des sujets cognitifs. La culture de participation aux changements et la formation pour le rôle d'acteur social sont peu représentés. Un élargissement des nouvelles compétences et du nouveau rôle des enseignants s’impose.

La socialisation professionnelle des débutants est souvent déficitaire. Le choc de la réalité est un phénomène toujours présent en début de carrière. Cette période d'adaptation aux réalités scolaires est souvent appelée "socialisation professionnelle des débutants ». C'est une période nécessaire et bénéfique pour les débutants si elle se passe bien. Le directeur et les collègues en ont la responsabilité. Malheureusement, dans bien des cas, les débutants entrent dans une culture de conformisme et sont socialisés pour s'adapter aux normes et les respecter, éviter les initiatives, etc.

Beaucoup d'enseignants considèrent que leur rôle principal est d'appliquer les décisions prises par ceux qui ont le rôle de décideurs. Ils sont passifs. Il y a du travail à faire pour éliminer ces stéréotypes. Ils souffrent d'un certain manque d'informations sur les différents changements proposés par la réforme scolaire, ce qui fait que leur perception de la réforme est parfois distordue.

Les autorités scolaires s'appuient notamment sur l'inspection pour introduire les changements. Même si ses objectifs et ses modes de travail ont été redéfinis, elle reste encore trop centrée sur le contrôle de l'exécution des directives et très peu sur son rôle constructif d'optimisation de l'activité, de stimulation des comportements créatifs et participatifs. 
Il reste donc à concevoir le champ scolaire comme un espace d'actions concrètes et d'interactions entre différents acteurs - enseignants, élèves, gestionnaires, parents, etc. C'est dans ces conditions que le rôle d'acteur des enseignants aura toutes les chances de s'affirmer de plus en plus.

\section{Bibliographie}

DUBAR Cl. (2000): La socialisation, Éd. Armand Colin, Paris.

FELOUZIS G. (1997): L'Efficacité des enseignants, PUF, Paris.

FERREOL G., NECULAU A., PAUN E. (2003): «The communication society: a challenge for education ", in: Learning and teaching in the communication society, Conseil de l'Europe, Strasbourg.

HETU J.-Cl., LAVOIE M., BAILLAUQUES S. (eds), (1999): Jeunes enseignants et insertion professionnelle, Éd. De Boeck, Paris, Bruxelles.

MIROIU A. (coord.), (1998): Invatamantul romanesc azi. Studiu de diagnoza. (L'enseignement roumain d'aujourd'hui. Étude de diagnostic), Éd. Polirom, Iasi.

NECULAU A. (coord.), (1997): Campul universitar si actorii sai (Le champ universitaire et ses acteurs), Éd. Polirom, Iasi.

OECD (2000), Analiza politicilor nationale in domeniul educatiei : Romania (Analyse des politiques nationales en éducation : Roumanie).

PAUN E. (1999): Scoala. Abordare sociopedagogica (L'École. Approche sociopédagogique), Éd. Polirom, Iasi.

PAUN E., POTOLEA D. (dir), (2002): Pedagogie. Fundamentari teoretice si demersuri aplicative (Pédagogie. Fondements théoriques et demarches appliquées), Éd. Polirom, Iasi.

VAN ZANTEN A. (dir), (2000): L'École. L'état des savoirs, éditions la Découverte, Paris. 


\title{
Les professeurs se battent-ils contre des moulins à vent?
}

\author{
Juan Carlos Gonzalez Faraco
}

Les politiques éducatives réformatrices de la seconde moitié du $\mathrm{XX}^{\mathrm{e}}$ siècle en Europe - dans l'Espagne des vingt-cinq dernières années - ont plébiscité le rôle prépondérant des professeurs comme collaborateurs nécessaires à tout changement éducatif. Il suffit de consulter la documentation internationale, comme le fameux rapport Delors de 1996 ou celui plus récent d'Eurydice $(2004)^{1}$ sur les enseignants européens. Dans le prologue, V. Reding, commissaire européenne, n'hésite pas à dire que les "professeurs sont des acteurs clés de toute stratégie qui vise à développer la société et l'économie». D'après A. Nòvoa ${ }^{2}$, ce type de discours politique contraste avec la pauvreté chronique des décisions pratiques et, par conséquent, donne une image fausse de la profession enseignante.

\section{Le malaise des enseignants}

La tentation de fabriquer des images autour de cette profession est par ailleurs très courante. Les professeurs, bien évidemment, créent leurs propres images, bien qu'elles soient beaucoup moins favorables que celles des hommes politiques. La majorité d'entre eux ont tendance à se voir comme des Don Quichotte qui luttent, quasiment en pure perte, contre une machine bureaucratique ou académique qui, depuis ses bureaux situés à une distance respectable des établissements, promeut des réformes purement théoriques. Loin de considérer qu'ils en sont les protagonistes ou les participants, ils se voient comme les grands oubliés du système. Beaucoup se sentent abandonnés à leur triste sort dans un univers scolaire raréfié, où ils naviguent à contre courant, avec la nostalgie des temps heureux où la paix régnait dans les salles de classe. Ils ne voient qu'une issue au chaos actuel : le retour à l'ordre et à l'autorité, et le rétablissement de leur fonction traditionnelle de spécialistes respectables d'une discipline. La réforme scolaire la plus récente en Espagne (Loi de qualité de l'éducation, 2002), lancée par le gouvernement conservateur du parti populaire, s'est servie du mécontentement du corps enseignant pour justifier certains changements dans le premier cycle du second degré (éducation scolaire obligatoire),

1. EURYDICE (2004). Key Topics in Education in Europe. Vol. 3. The teaching profession in Europe: Profile, trends and concern (http://www. eurydice.org).

2. Nòvoa, A. (1999). «La nueva cuestión central de los profesores. Exceso de discursos, pobreza de prácticas». Cuadernos de Pedagogía, $\mathrm{n}^{\circ}$ 286, pp. 102-108. 
celui qui pose le plus de problèmes dans le système éducatif ${ }^{3}$. Les mesures prises favorisaient les parcours différenciés des élèves par la création d'itinéraires et de branches diversifiées avant la fin de l'enseignement obligatoire, renforçaient le pouvoir du chef d'établissement et durcissaient le système d'évaluation.

Il faut rappeler que dans l'élaboration de cette loi, très contestée par l'opposition mais en général bien accueillie par les professeurs, les résultats d'une évaluation générale du système ${ }^{4}$, confiée à un groupe d'experts par le ministère de l'Éducation, et ceux de la première enquête PISA de l'OCDE (2000) qui donnait une image médiocre des élèves espagnols, ont joué un rôle important. La publication de la deuxième enquête (2003), dont les résultats sont encore pires, et la suspension par le nouveau gouvernement socialiste de la Loi de qualité ont relancé le débat, abondamment relayé par les médias, sur le faible rendement et le manque de motivation des élèves, sur les conflits dans les classes et le rôle ingrat des enseignants.

La nouvelle équipe ministérielle de gauche, avant de se lancer dans sa propre réforme, a diffusé sur Internet un document pour nourrir le débat, intitulé «Une éducation de qualité pour tous et partagée par tous » ${ }^{5}$, qui se résumait à un inventaire de bonnes intentions. Naturellement, le document insistait sur le «rôle indispensable» des professeurs dans le processus réformateur imminent dont les objectifs sont semblables à ceux que l'on retrouve invariablement dans tous les forums internationaux : améliorer la formation initiale, créer un statut professionnel, faciliter la mobilité professionnelle, améliorer la reconnaissance sociale, etc. Comme on pouvait le prévoir, le nouveau projet de Loi organique de l'éducation (LOE, 2005), promu par les socialistes, consacre un long chapitre aux professeurs et à une meilleure reconnaissance sociale de la fonction enseignante. C'est avec une froideur et une circonspection manifestes que les enseignants accueillent ces «nouveaux» discours, d'autant que les réformateurs actuels ont repris, en partie du moins, le modèle pédagogique promu par les précédents gouvernements socialistes dans les années quatre-vingts et quatrevingt-dix ${ }^{6}$. Les critiques émises à l'époque par de nombreux professeurs, surtout dans le secondaire, contre ce modèle global et constructiviste, sont toujours d'actualité même si les enseignants se montrent sans doute aujourd'hui moins agressifs. Pour beaucoup, cet apaisement n'a d'autre explication que l'acceptation résignée d'une situation professionnelle qui demeure pénible. C'est le syndrome du «burn out» du professeur.

Les récits que les enseignants font de leur profession au quotidien dépeignent un paysage désolé dans lequel se joue un drame avec des «victimes» - eux-mêmes - et des «bourreaux» : des «objecteurs scolaires» qui ne veulent pas

3. Vicente Algueró, F. de (2002). «El profesorado en la Ley de Calidad». Revista de Educación, n 329, pp. 67-76.

4. Ministerio de Educación, Instituto Nacional de Calidad y Evaluación (1998). Diagnóstico del Sistema Educativo. La escuela secundaria obligatoria. La profesión docente (vol. 5).

5. http://www.mec.es

6. Ley de Ordenación General del Sistema Educativo (LOGSE, 1990). 
apprendre et créent des problèmes de cohabitation; des parents qui se désintéressent de l'éducation de leurs enfants ou qui envahissent leur espace professionnel; des experts qui les utilisent sans ménagement pour leurs expériences pédagogiques $^{7}$; des hommes politiques et une administration qui ne respectent pas leur autonomie professionnelle. Cette vision alimente ce que l'on appelle le "malaise enseignant», un concept assez vague né d'études sur la personnalité et la santé des professeurs. À ces perceptions plus ou moins subjectives viennent s'ajouter de vieux problèmes et des revendications qui n'ont jamais abouti ainsi que quelques indicateurs sociologiques préoccupants ${ }^{8}$ : vieillissement du corps professoral, future pénurie de professeurs, féminisation croissante, prolétarisation ${ }^{9}$ ou professionnalisation à moitié réussie seulement, perte de prestige social, bas salaires, formation inadaptée ${ }^{10}$, surtout dans le secondaire ${ }^{11}$, etc. Les avis divergent quand il s'agit d'en déterminer les causes et les responsabilités, bien qu'on ait tendance le plus souvent à désigner comme principaux responsables divers agents parmi lesquels ne figurent généralement pas les enseignants.

Cependant, des analystes qui adoptent une position tout à fait différente voient dans le "malaise» des professeurs un de ces discours dont ils se servent pour défendre leurs privilèges professionnels. Le sociologue espagnol M. Fernández Enguita (1996), dans un article ${ }^{12}$ qui fit grand bruit lors de sa parution, mentionne la résistance opiniâtre des enseignants aux tentatives d'innovations de l'administration. Il en conclut que les progrès nécessaires du service public d'éducation se heurtent de front au pouvoir, aux intérêts et aux habitudes du corps enseignant ${ }^{13}$. Le recours à la «victimisation» ou à d'autres tactiques défensives ne serait que l'expression d'un corporatisme masqué. D’après lui, il existe trois causes à cette tentative de "privatisation » corporative d'un espace public tel que l'école : la féminisation de la profession, le manque de vocation des enseignants et l'irresponsabilité accommodante des syndicats.

7. Ruiz Paz, M. (2003). La secta pedagógica. Madrid, Grupo Unisón de Ediciones.

8. Pérez Gómez A. et al. (2003). "La situación profesional de los docentes. Luces y sombras». Cuadernos de Pedagogía, $\mathrm{n}^{\circ}$ 326, pp. 34-40.

9. Arenas Vázquez C. (2001). «El profesorado de secundaria frente a las reforma ». Cuadernos de Pedagogía, $\mathrm{n}^{\circ} 303$, pp. 84-86.

10. Ph. Perrenoud argumente en faveur d'un modèle de professionnalisme ouvert pour la fonction enseignante, et par voie de conséquence pour la formation, par opposition au modèle traditionnel de compétence minimum qu'adoptent, dans leur grande majorité, les enseignants du secondaire comme modèle professionnel de référence. Voir « La profesión docente, entre la proletarización y la profesionalización ». Perspectivas, vol. 26, n 3, pp. 549-570, 1996.

11. Par exemple, en ce qui concerne la France, A. Van Zanten souligne le manque de cohérence de la formation continue des professeurs du secondaire. Voir "La profession enseignante en France, quelles évolutions?», Revue internationale d'Éducation Sèvres, $n^{\circ}$ 30, p. 90.

12. Fernández Enguita, M. «Es pública la escuela pública». Cuadernos de Pedagogía, n² 284, pp. 76-81. Voir aussi : «Las profesiones como poder social: el caso del profesorado ». Revista de Educación, n 324, pp. 143-154, 2001.

13. Le fait que les enseignants espagnols soient majoritairement des fonctionnaires ( $74 \%$ ) est essentiel pour comprendre les attitudes défensives des professeurs face à d'autres acteurs du système. 


\section{Une redéfinition \\ professionnelle nécessaire}

Pourtant, que l'on considère les enseignants comme des Don Quichotte ou des Sancho Pança, il est intéressant de relever qu'une bonne partie des analyses actuelles tournent autour de la nécessité de redéfinir leur identité. Les arguments utilisés peuvent parfois paraître presque métaphysiques voire anachroniques. Ainsi, dans un bref et récent article intitulé "Être un maître » ${ }^{14}$, une écrivaine célèbre affirme que l'enseignement n'est pas une profession mais un métier, car enseigner ne revient pas à "exercer» une profession mais bien à vivre une "passion». Dans ce contexte, on ne s'étonnera pas non plus (même si cela ne laisse pas d'inquiéter) que le psychiatre espagnol C. Castilla del Pino ait utilisé l'expression toute faite "pathologie de l'identité» pour décrire les problèmes de santé les plus fréquents de ses nombreux patients enseignants. D’une façon ou d'une autre, la nécessité de redéfinir la profession enseignante est devenu l'objet prioritaire d'un débat éducatif non dénué de rhétorique et parcouru à l'heure actuelle par des chemins à première vue discordants.

Certains mettent leur espoir dans les nouvelles technologies comme voie de pénétration des innovations didactiques et comme outil de revitalisation de l'école et de ses acteurs. De fait, les nouvelles technologies de l'information et de la communication sont le secteur le plus demandé par les professeurs en formation continue et le plus développé dans les programmes de l'administration destinés à améliorer le système éducatif. Pour beaucoup, le jeu technologique serait le répulsif dont l'École et la profession enseignante auraient besoin après une étape agitée de réformes hautement idéologiques. D’autres, en revanche, parient sur la revitalisation morale et professionnelle du professeur avec l'adoption de mesures améliorant ses conditions de travail et son prestige social, mais aussi grâce à un appel, presque poétique, à l'optimisme ${ }^{15}$ et à l'engagement éthique. Le succès d'un film français comme Être et avoir met en évidence le désir, partagé par un grand nombre, d'affronter le problème de l'école actuelle en prenant la défense de ces valeurs considérées comme désuètes par d'aucuns, plutôt qu'en ayant recours à des innovations techniques ou pédagogiques.

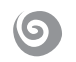

C'est dans ce labyrinthe de discours et d'images superposés qu'évolue aujourd'hui la fonction enseignante. De réforme en réforme, a peu à peu été défini le type de professeur dont on ne veut pas, même si c'est avec quelques désaccords. Il est urgent de nous mettre d'accord maintenant, sur le type de professeur dont nous avons besoin, mais surtout sur les décisions, probablement radicales, qu'il faudra prendre pour le «fabriquer». Ces décisions seront-elles effectivement prises un jour?

14. Aldecoa J. (2003). «Ser maestro», Cuadernos de Pedagogía, $n^{\circ}$ 326, p. 41.

15. Santos Guerra Miguel A. (2004). «Invitación al optimismo ». Cuadernos de Pedagogía, nº 334, pp. 86-90. 


\section{Suisse}

\section{Professionnels, oui. Mais intra muros.}

\section{Danièle Périsset Bagnoud}

Plusieurs initiatives ont tenté, et tentent encore, de transformer le système d'éducation suisse et, notamment, de faire avancer le processus de professionnalisation de l'enseignement. Plusieurs catégories d'acteurs y participent : les acteurs politiques, qui ont proposé, au cours de ces dix dernières années, différents cadres législatifs propres à favoriser notamment l'accroissement de l'autonomie des établissements et à inscrire les plans d'étude dans une perspective longitudinale inédite, les acteurs professionnels, par leurs associations impliquées dans les réformes politiques; les acteurs de la recherche en éducation, qui fournissent aux précédents des fondements scientifiques sur lesquels se basent les propositions de réformes.

\section{De la professionnalisation}

Cependant, si l'on trouve, à l'intérieur même de chacune de ces catégories d'acteurs, diverses tendances qui nourrissent bien des paradoxes autour de l'objet "professionnalisation de l'enseignement», les positions que prennent les enseignants, individuellement ou par leurs associations, ne sont pas les moins étonnantes.

Le terme de professionnalisation est aujourd'hui couramment utilisé. Pourtant, il suscite bien des débats. Ces qualités font cependant consensus : l'enseignant professionnel est au bénéfice d'une formation, longue et spécialisée, de niveau supérieur et qui relève de savoirs scientifiques; il réalise en autonomie des actes intellectuels et non routiniers qui engagent sa responsabilité; le corps professionnel est structuré en organisme officiel auto-régulé et fort d'une reconnaissance sociale élevée.

C'est la seconde caractéristique qui retient à ce jour le plus l'attention des associations d'enseignants. Autonomie et responsabilité sont les maîtres mots des discours relevant des évolutions des systèmes scolaires et se déclinent sur toute la gamme de leurs possibles interprétations. Paradoxes et ambiguïtés nourrissent les positions des enseignants que révèlent deux documents récents : le Code de déontologie du Syndicat des enseignants romands (2004) et les résultats d'une enquête menée en 2002 auprès de onze mille enseignants en Suisse alémanique par leur association professionnelle ( $\mathrm{LCH})$. 


\section{Autonomes et responsables, mais dans les murs de la classe}

Une lecture attentive des déclarations d'intention du Code de déontologie révèle la trame des difficultés. Ainsi est souhaitée une lecture critique et responsable de ce à quoi est exposé l'enseignant ( «faire preuve de sens critique, d'autonomie et savoir prendre ses responsabilités»). Aucune trace de collaboration/ partenariat/négociation éventuels avec la hiérarchie ou les pairs n'apparaît. À quel implicite, et à qui, rattacher ce sens critique, sinon à celui que peut mettre chaque acteur, d'après ses expériences et stratégies personnelles? Pourtant, point trop de liberté ne faut : un bon professionnel tient «compte des options pédagogiques de son association professionnelle». Plus loin, une norme intériorisée, puisque non définie, est donnée pour évidente et partagée : "faire preuve de conscience professionnelle». Cette terminologie désuète trahit la persistance de l'origine normalienne des enseignants. La professionnalisation donnée à voir ici reste confinée dans les murs de la classe, s'y gonfle d'une autonomie toute relative et ne s'aventure guère au dehors, où sévit un environnement public, politique, dangereux.

L'enquête individuelle auprès des enseignants confirme ce sentiment de repli. Elle révèle la permanence du lieu commun lié à la nostalgie d'un passé mythique d'avant la démocratisation-massification des études, lorsque les publics étaient clairement sélectionnés selon leur provenance sociale et ne posaient, de ce point de vue, guère de problème. L'environnement social et éducatif est vu comme déficitaire; les initiatives de la hiérarchie sont rejetées (excès de réformes et absence de consultation) mais, le paradoxe n'est pas le moindre, le manque de soutien et de directives de la part de ces mêmes autorités est déploré. L'individu s'adapte apparemment aux changements mais dans son for intérieur, il se rebelle et en appelle à l'ancien ordre social. L'enseignant veut être professionnel, certes. Mais dans le cadre de sa classe, dans son jardin protégé et sous le regard «bienveillant» et paternel d'une hiérarchie qui le guide.

\section{Acteurs, mais insoumis}

Entre les intentions déclarées des systèmes éducatifs et celles qui leur sont prêtées, entre les stratégies des associations d'enseignants et celles des enseignants, il y a tout un jeu de stratégies que nourrissent les enjeux spécifiques des différents groupes d'acteurs et que sous-tendent les cultures collectives issues de l'histoire longue des mentalités corporatives. Leur identité ne se modifie pas par décret. La transformation des systèmes éducatifs accompagne étroitement le développement social, qui marque lui-même l'évolution des formations d'enseignants. L'histoire de l'école, en perpétuelle évolution, est faite de tensions et de ruptures, de permanences, de tentatives de changements et de résistances. C'est une évidence souvent oubliée. Et l'évolution est mue par les conflits entre les groupes d'acteurs sociaux. 
Les enseignants, assurément, sont acteurs de l'évolution des systèmes scolaires, mais dans «leur» sens, celui qui répond le mieux à leurs besoins, à leurs stratégies. Leur apparente résistance est peut-être le moyen qu'ils privilégient pour dire leur difficulté à entrer dans un jeu d'enjeux dans lesquels ils ne se reconnaissent pas mais dans lequel ils voudraient qu'on leur donne la place, ultime paradoxe, qu'ils ne savent, ne peuvent, ne veulent peut-être pas encore conquérir. 


\title{
Angleterre
}

\section{Un mythe professionnel en souffrance}

\author{
Régis Malet
}

Les enseignants anglais, en référence à des professions établies, affirment traditionnellement leur professionnalisme par leur influence en matière de choix des contenus d'enseignement et par l'affichage d'une éthique et d'une responsabilité professionnelle élargie, garantes d'une position sociale reconnue et d'une autonomie d'exercice.

\section{La crise de confiance}

Ce professionnalisme sur lequel le corps enseignant britannique a construit son identité et sa légitimité n'a de validité que si le politique, et la société elle-même, lui accordent crédit et confiance. Or ce contrat moral entre la nation et les enseignants semble aujourd'hui défaillant. Depuis les années 1980, les gouvernants britanniques, de la Nouvelle Droite (New Right), puis de la Nouvelle Gauche (New Labour), ont repris le pouvoir sur l'école et un corps enseignant qui, du fait de son histoire et de ses formes de légitimation, est moins protégé que ne l'est le monde enseignant français par un lien organique entre l'École, les maîtres et l'État.

La «désuniversitarisation» de la formation, la standardisation des compétences professionnelles, l'élargissement et la technicisation des tâches, l'imputabilité des enseignants (accountabiliy), sont autant d'indicateurs de la mise en faillite du mythe professionnel et de l'idéal d'autonomie dont il était porteur. Cette crise de confiance et ses conséquences en termes de procéduralisation du travail, auxquels se conjuguent les difficultés, communes avec la France, liées à la diversification des publics scolaires dans un enseignement de masse, engendrent chez les enseignants britanniques des doutes identitaires et des incertitudes sur leur capacité à assumer les missions qu'on leur assigne ${ }^{1}$.

\footnotetext{
1. Si la plupart des nations européennes sont exposées à des risques de pénurie d'enseignants, la GrandeBretagne (à l'exception notable de l'Écosse), est parmi les pays les plus touchés par cette désaffection pour la profession et par les décisions d'abandon au cours des premières années dans le métier (Commission Européenne, 2004 ; Leclecq, 2003). Les mesures d'incitation par des primes d'accueil offertes aux néo-enseignants (le Golden Hello), ne suffisent pas à combler cette pénurie, à tel point que des formations alternatives ont été introduites. Le Graduate and Registered Teacher Programmes (GRTP) en Angleterre et au Pays de Galles est une nouvelle voie de formation en emploi qui permet aux établissements de recruter des personnes non qualifiées pour l'enseignement et de les former dans un programme de formation individuel qui mène au Qualified Teacher Status (statut d'enseignant qualifié).
} 


\section{Des évolutions contradictoires}

Sur un plan politique, les indices de ces évolutions se déclinent selon un double mouvement, de globalisation et de fragmentation des politiques scolaires : renforcement de l'autonomie (maîtrisée) des établissements, contractualisation des objectifs éducatifs et développement du management participatif, déconcentration du pouvoir hiérarchique, individualisation des carrières des personnels enseignants, accroissement du pouvoir parental (Gewirtz, Ball \& Bagley, 1995), passage d'une logique administrative de gestion des personnels, à une logique managériale de gestion des ressources humaines (Laderrière, 2004), développement d'outils d'évaluation des élèves, des enseignants et des établissements (Broadfoot, 1996; Lessard \& Meirieu, 2005). Ces diverses évolutions, observables dans la plupart des pays européens selon une intensité variable, sont largement réalisées en Angleterre.

Singulièrement, dans un pays de tradition communautariste dans lequel la culture organisationnelle a pénétré l'univers scolaire bien longtemps avant la France, on voit se développer des formes de régulation du travail scolaire hybrides, marquées à la fois par un modèle libéral conférant aux établissements une marge d'autonomie importante dans le recrutement et les carrières de leurs personnels et dans leurs liens avec l'environnement social et économique, et par un modèle bureaucratique plus récent, l'État s'accordant un rôle de contrôle renforcé des établissements et des personnels qui y exercent. Responsables jusqu'alors auprès de leur employeur, l'établissement et les autorités éducatives locales, les écoles et les enseignants le sont dorénavant auprès de l'État (Malet \& Brisard, 2005). Ces évolutions voient émerger des formes d'administration scolaire promouvant des notions managériales, singulièrement marquées par des préoccupations éthiques - school improvement, school effectiveness, educational and management - en conformité avec les principes qui animent le New Labour (associant réalisme économique, souci d'efficacité et justice sociale).

Au-delà de ce diagnostic global et programmatique, l'objectif est aussi d'examiner comment les évolutions récentes des conditions d'exercice et les «injonctions au changement» des pratiques transforment le métier d'enseignant, compte tenu de cultures professionnelles, organisationnelle et de formes d'accompagnement spécifiques. L'un des enjeux est également d'examiner les formes de leadership observables, dans les établissements scolaires britanniques, par des administrateurs qui sont les interfaces décisives entre les incitations institutionnelles et les enseignants. Il est enfin de mettre en perspective les formes d'accompagnement, de responsabilisation et de reconnaissance auxquelles donnent lieu ces velléités d'élargissement des missions et du travail des enseignants.

En Angleterre comme dans d'autres pays européens, les transformations des formes d'administration de l'école et des enseignants attestent d'une ambition 
claire : il s'agit d'assurer une autorité effective de l'autorité macro-politique avec un État fort, tout en accordant une autonomie de gestion au niveau micropolitique des établissements : au prix d'un affaiblissement du potentiel des autorités éducatives locales, devenu embarrassant, et de la promotion d'un modèle de conformation professionnel promoteur, pour ce qui est du travail des enseignants, de ce que j'appellerais un «différentiel normé». Il semble que le politique ouvre à combiner une norme professionnelle locale plus ou moins sédimentée, avec la tendance internationale au développement de formes de pilotage scolaire fondées sur la rationalisation et la responsabilisation individuelle et collective.

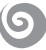

Il convient probablement d'interroger, en le plaçant sur un plan international, ce double processus de rationalisation/responsabilisation du travail des enseignants, dont l'aboutissement visé est le développement d'une culture du collectif, d'un professionnalisme collectif. Il vise à permettre, à terme, la mutualisation et l'harmonisation des ressources et des pratiques propres à une communauté éducative, dans une perspective de rationalisation collective de l'offre de formation. De ce point de vue, la promotion de l'organisation scolaire apprenante à l'œuvre depuis la dernière décennie trouve matière ici et là, avec l'introduction de nouvelles formes de management social des établissements, et en particulier dans le contexte anglais, à armer les réformes éducatives en cours d'un arsenal de «technologies de gouvernance» (Ball, 2003) qui en assurent la mise en ouvre locale.

\section{Bibliographie}

BROADFOOT P. (1996): Education, Assessment and Society, Buckingham: Open University Press.

GEWIRTZ S., BALL S. \& BOWE R. (1995): Markets, Choice and Equity in Education, Buckingham: Open University Press.

LADERRIERE P. (2004): La Gestion des ressources humaines dans l'enseignement : où en est l'Europe?, Paris : L'Harmattan.

LESSARD C. \& MEIRIEU P. (Dir.) (2005): L'obligation de résultats en éducation. Évolutions, perspectives et enjeux internationaux, Bruxelles : De Boeck.

MALET R. \& BRISARD E. (Dir.) (2005): Modernisation de l'école et contextes culturels. Des politiques aux pratiques en France et en Grande-Bretagne, préface d'Agnès van Zanten, Paris : L'Harmattan. 


\title{
France
}

\section{Les enseignants, spectateurs ou acteurs responsables?}

\begin{abstract}
Alain Bouvier
Nous évoquons le métier d'enseignant, en France, par le biais de l'évaluation de l'action pédagogique des professeurs. Nous formulons cinq constats nationaux, puis nous pointons six paradoxes probablement plus internationaux.
\end{abstract}

\section{Quelques constats}

En France, le système éducatif fonctionne avec une importante division des tâches (d'inspiration taylorienne) : entre les registres pédagogique et administratif; entre le pédagogique et la vie scolaire; entre les cours d'enseignement général et d'enseignement professionnel; entre ceux qui enseignent, ceux qui dirigent et ceux qui les évaluent.

L'évaluation des enseignants exerce un fort pouvoir, un peu mythique. Elle est assurée par un corps spécialisé : les inspecteurs pédagogiques. Selon le discours dominant du milieu enseignant, cette évaluation jouerait un rôle important par rapport à leurs pratiques pédagogiques. Les inspecteurs sont quasiment les seuls adultes témoins (tous les cinq à dix ans) de fragments de celles-ci. Ils exercent ainsi un pouvoir symbolique symétrique de celui de l'enseignant qui enseigne la porte fermée. Qu'en est-il, réellement, de ce pouvoir pédagogique de l'inspection? Mythe ou réalité?

L'évaluation des enseignants repose sur l'observation, très brève et espacée dans le temps, de leurs pratiques pédagogiques par leur inspecteur. Les enseignants sont préalablement prévenus de cette venue. Pourtant les recherches nous apprennent qu'il faut près d'une semaine d'observations outillées pour connaître la pratique pédagogique réelle d'un enseignant. Et n'existe aucun rapport à l'autoévaluation, Or, on sait, qu'il existe un écart considérable entre ce que les enseignants croient pratiquer et ce que révèlent les observations lourdes. Enfin, Claudine Peretti, directrice de la direction de l'Évaluation et de la prospective au ministère de l'Éducation nationale (DEP), fait observer que l'on ignore pratiquement tout des relations entre pratiques pédagogiques et résultats des élèves. Alors, faut-il continuer ainsi à tout baser sur de telles observations?

En France, l'évaluation externe ne peut en rien s'appuyer sur des autoévaluations conduites par les équipes pédagogiques : elles n'existent pas. Aucune méthodologie n'est envisagée, ni proposée. Faut-il le faire? Le préconiser, puis 
se diriger vers un «contrôle des contrôles» qui vérifierait l'existence d'une procédure formalisée? Et si celle employée est bien celle décrite? Une telle perspective modifierait profondément le rôle des inspecteurs. Le souhaitent-ils? Sont-ils prêts à de telles évolutions?

Afin de se diriger vers une approche plus globale et collective de l'évaluation de l'action pédagogique, pour dépasser la seule évaluation ponctuelle des individus, l'établissement scolaire pourrait imaginer une évaluation interne responsabilisante. En d'autres termes, il pourrait concevoir un véritable dispositif d'autoévaluation du registre pédagogique et pour cela il pourrait faire appel à des experts (en France, il n'existe pas d'ordre des enseignants). Pour croiser approches individuelles et approche collective. Pour mieux connaître et comprendre les «effets maîtres» et les «effets d'établissement»; voire les «effets discipline", s'il en existe. Et s'inscrire dans une perspective d'un benchmarking basé sur des performances mesurées. Sinon, c’est la «BLOG-évaluation» qui développera le pouvoir qu'elle commence déjà à exercer.

\section{Quelques paradoxes}

Alors que les enseignants ont une haute qualification universitaire, d'au moins cinq ans, de "cadres », comparable à celle des ingénieurs ou des architectes, pourquoi (premier paradoxe) acceptent-ils de s'enfermer dans une organisation aussi taylorienne et cloisonnée? Qu'y gagnent-ils? Notamment en termes d'image et de reconnaissance sociale? Ne seraient-ils «cadres» que pour des fractions de leur temps?

On n'a jamais eu autant d'informations sur l'École et sur ses résultats. Sur «l'effet maître» et sur «l'effet établissement». Sur les taux de réussite aux examens : taux bruts, taux par rapport aux populations d'élèves accueillis en seconde, par rapport aux catégories socioprofessionnelles des parents (avec la notion de «taux de réussite attendus»). Mais aussi sur la plus-value des établissements, sur l'évolution de celle-ci, sur l'insertion professionnelle des élèves, etc. Le grand public est avide de telles informations diffusées par les médias. Elles influencent même le prix des terrains fonciers et des locations. Paradoxalement, le milieu enseignant, lui, se tient à l'écart de ces données. Pourquoi se place-t-il, ainsi, dans une forme d'ignorance collective vis-à-vis de son action?

Les enseignants français sont des fonctionnaires de l'État, au sein d'un service public. Pourtant, ainsi, ils agissent en «libéraux individuels» de l'éducation. Pourquoi défendent-ils aussi fortement leur «autonomie» pédagogique ponctuelle, formelle, individuelle et d'apparence, sans, paradoxalement, en assumer les responsabilités collectives? Notamment sans se doter d'un système d'autoévaluation?

Pourquoi, en France, l'évaluation de l'enseignement, présente dans tous les discours, est-elle si lourde au niveau des symboles et si légère pour ses conséquences : c'est-à-dire quasiment nulles? 
Les enseignants exercent «la profession du savoir». Mais, paradoxalement, ils ne capitalisent pratiquement pas sur «le savoir de leur profession». Pourquoi n'entrent-ils pas dans une logique de régulation collective de leur action pédagogique? Pourquoi n'appliquent-ils pas à eux-mêmes, ni individuellement, ni collectivement, ce qu'ils préconisent pour les apprentissages de leurs élèves?

Dans beaucoup de pays, l'évaluation de l'enseignement suit un code de déontologie, se veut fidèle, valide et utile. Empreinte d'une certaine éthique professionnelle et de dignité. Elle se réfère au registre de la morale civique, comme pour des animateurs de colonies de vacances. En France, pourquoi élude-t-elle le registre professionnel : les résultats, l'intérêt des élèves, de leurs familles, du système social et économique? Qui se préoccupe des résultats? De l'efficacité de l'action des enseignants? De son efficience? Des performances réalisées?

\section{Amorce de perspective}

On observe une coupure croissante et inquiétante entre une culture managériale des cadres (qui se développe) et une culture de la résistance du milieu enseignant. Si la profession enseignante décide d'ignorer les résultats de son action, à qui laisse-t-elle le champ libre? De facto, à qui délègue-t-elle sa responsabilité ? À la BLOG-évaluation? À la BLOG-régulation?

Se voulant responsable, elle ne peut continuer de la sorte. Peut-on décider de se diriger vers des systèmes apprenants? 